\title{
Pain disorder, hysteria or somatization?
}

\author{
Harold Merskey DM FRCP FRCPC FRCPsych
}

$\mathrm{P}$

Pain used to be a simple issue. It was caused by physical injury or disease and the sufferer had to rest and take opium. That was about two hundred years ago. A few scattered commentators from Jeremiah (Lamentations I:12-13) to Montaigne (1) had the idea that emotion could cause pain or influence it. The development of anatomical knowledge, closely followed by physiology and then pathology, produced a dilemma. There were many pains that could not be explained by the most modern physical methods of the nineteenth century. Hodgkiss (2) has tersely labelled the problem as "pain without lesion". The nineteenth century solution lay in a diagnosis of hysteria.

\section{Meanings of hysteria}

Hysteria was a handy term with multiple meanings including depression, stress reactions and some physical disorders attributable to cerebral disturbance, but it was not well described. By the end of the nineteenth century, a motivational explanation was also offered in Freudian theory. A simplified version of the latter was then used by doctors to account for the combat neuroses and anxiety disorders of the First World War (3). The problems of seemingly physical complaints without adequate organic evidence came to be generally defined as conversion disorders and were linked with psychological symptoms for which similar mechanisms were postulated, eg, cases apparently of 'psychogenic' loss of memory. The latter were called dissociative disorders and because of the similarity of the theories about the two groups, both were labelled under the term "Dissociative" in the International Classification of Diseases, 10 th revision (4).

The word conversion was originally used because Sigmund Freud (5) had thought that the state of feeling, anxiety or depression, which he called affect, and which was connected with a psychological conflict, might be directly converted from a sort of chemical status within the body to a physical paralysis. It later lost that meaning and simply reflected bodily symptoms - apparently caused by conflict and not by overt emotional change.

\section{Conversion disorder}

Typically, in the literature of the twentieth century and to date, conversion disorder has been diagnosed on the following grounds:

1. The absence of appropriate physical illness.

2. The presence of signs which demonstrate that the patient thinks that he or she cannot do something when they can. The standard example of this is Hoover's test where the patient reports inability to move a limb and shows no power when asked to press the heel down on a couch or lift it in the air, but is shown to have the power to press down on a hand when the opposing leg is actively elevated. More dramatically, Figure 1, a drawing by Charcot (6), shows the patient doing more than a normal person could do, while still complaining of an inability to walk normally.

3. There should also be evidence of an emotional problem which is resolved, at least temporarily, by the production of the symptom and of which the patient appears to be, either to some extent or wholly, unaware.

4. The diagnosis is finally supported if adequate therapy or management of the supposed problem or the external environment succeeds in 'curing' the symptom and the patient is satisfied with this explanation.

This condition is not always recognized, but follows from the theory of psychoanalytic treatment and of the original diagnosis of hysteria. If the symptom is due to an idea or 'repressed complex', the idea has to be brought to light and its origins and effects resolved. Theoretically, this should be

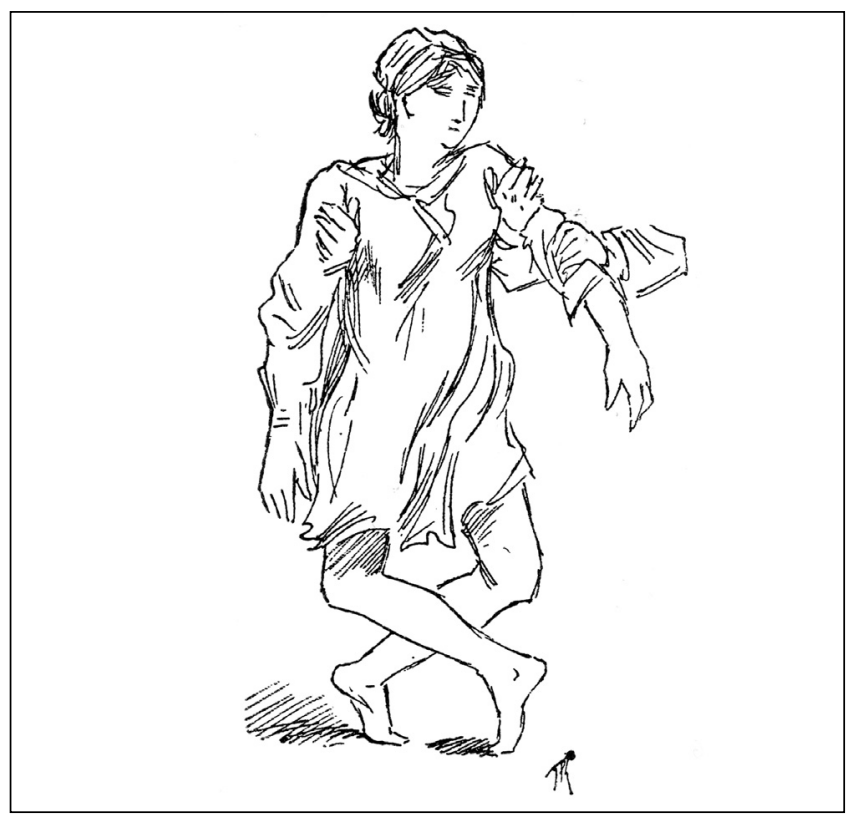

Figure 1) A sketch by JM Charcot. Reproduced from reference 6 
achieved by free association, because to tell the patient what one takes to be the reason amounts to providing a suggestion that destroys proof of the condition being truly unconscious and recovered. It happens that Freud himself frequently appears to have violated this condition (7). However, the theory implies that the patient will come to recognize and acknowledge a source for the symptoms without suggestion. As Jones (8) puts it:

"...experience has amply demonstrated that when...[repression is reversed]...the abnormal manifestation or symptom ceases. The central aim of the psychoanalytic method, therefore, consist in enabling the patient to discover and appreciate the significance of the process that manifests itself as a symptom."

These fundamental phenomena all refer to conversion symptoms which may be a loss of either a motor function or a sensory function. In the case of some sensory functions, positive evidence can still be found, eg, using an optokinetic drum in patients complaining of complete blindness. However, in general, apart from ocular symptoms, it is usually harder for the neurologist or relevant specialist to demonstrate sensory contradictions that clearly show persisting competence on the part of the patient than it is to demonstrate motor ones, although nerve conduction studies and cerebral evoked potentials may help.

\section{Diagnosis of hysterical pain or dissociation}

Pain is hard to prove as a hysterical symptom. It can probably only be partly shown by demonstrating that there is a major emotional problem and that the pain goes away when the problem is resolved. At the conclusion of treatment, one would also need the agreement of the patient to the idea that the symptom was solving a particular problem. Even that lacks the nice empirical and conceptual strength of demonstrating movements that the patient maintains are impossible.

Supposed dissociative disorders, eg, some memory disturbances, are also somewhat harder to prove than conversion disorders, but are still somewhat more susceptible to identification and characterization than hysterical pain - if it exists.

\section{Classification: American Psychiatric Association}

We can now look at how different diagnostic systems have attempted to characterize pain arising from the patient's idea of having pain, when the patient is not psychotic.

The most detailed systematic approach is that of the Diagnostic and Statistical Manual (DSM) of Mental Disorders of the American Psychiatric Association in its third (DSM-III) (9), third revised (DSM-III[R])(10) and fourth editions (DSM-IV) (11). These three versions have undergone successive modification for the diagnosis of somatoform pain, mainly in an attempt to cope with the issue of alleged 'psychogenic pain'. A little preliminary comment is necessary to sketch the major change that first appeared in DSM-III.

Previous versions of psychiatric classification systems had provided categories of hysteria which were rather vague in general, and also looked at issues of personality connected with socalled hysterical symptoms. There was much dissatisfaction with the diagnosis of hysteria because, in practice, it frequently appeared to be misleading and was often confused with an organic disorder $(3,12)$. Whether or not the patients had an internal conflict, was a fertile source of arguments between psychiatrists and neurologists, and within the two disciplines as well.

A group of American investigators (13) decided that they would identify 'hysteria' as only those persons who had multiple symptoms and no relevant physical disorder demonstrated over a significant portion of their lives. While this failed to deal with the interesting theoretical issues, it provided a group of individuals (all or almost all women) who could be identified with reliable research diagnostic criteria and whose behaviour could be studied. Cohen et al (13) were correct, of course, in this, although their approach left dissatisfied those who were uneasy with the idea that one might cut off one end of a group on a continuum and simply study the severe end of it. Guze (14) summarized the main features as follows:

"First the patient must demonstrate a dramatic or complicated medical history beginning before age thirty-five. Second the patient must admit to twenty-five symptoms in nine of ten special review of symptoms areas. Third, no other diagnosis can be made to explain the symptoms."

This was then called Briquet Syndrome after Pierre Briquet, the nineteenth century French physician, who was considered to have described something similar, although that view is questionable (15).

The areas of symptoms included headaches; classical neurological symptoms such as blindness, paralysis, anesthesia, aphonia, fits etc; fatigue, a lump in the throat, fainting spells, visual blurring, weakness, dysuria; difficulties with breathing, weight and eating; abdominal complaints; dysmenorrhoea and other menstrual complaints; changes in sexual interest; pains in various parts of the body; and nervousness, fears and depressed feelings, etc.

Ordinarily, for a symptom to be counted towards the relevant number, the patient should have sought treatment for it from a physician or medicated herself for it. This group of criteria was later used in DSM-III under the heading "Somatization Disorder".

The original concept of somatization was put forward by Wilhelm Stekel, an early twentieth century psychoanalyst (16), and was codified, as it were, by Lipowski, who proposed a definition of somatization in 1968 and amended it in 1988 to read:

"The tendency to experience and communicate somatic distress and symptoms unaccounted for by pathological findings, to attribute them to physical illness, and to seek medical help for them" (17).

The idea of 'somatization' or 'somatizing' seems to have taken off from there.

Somatization disorder thus came into DSM-III and was placed in an overall group of conditions known as "Somatoform Disorders". The other diagnoses in this group reflected an attempt to define patients with hysterical symptoms (as they were formerly known), although the word hysteria was virtually banished from the volume, and the classifiers relied upon 'conversion disorders' and 'dissociative disorders' putting conversion disorders in the somatoform group, while dissociative disorders became a separate adjoining category.

Within the somatoform group, patients with classical hysterical symptoms, but not pain, were placed under 
"Conversion Disorder", while those with pain as a single symptom, or one or two pains, were classified as having "Psychogenic Pain Disorder". Yet another category, a 'rag bag' or catch-all category, was needed (as in all psychiatric and other medical classifications) and atypical somatoform disorder covered that matter.

The diagnosis of Psychogenic Pain Disorder in DSM-III owed something to notions of hysteria and something to concepts of psychological causes of pain that were in the literature in the 1970s. The criteria tended to resemble those that identified it conceptually with conversion disorder, apart, of course, from basing it on a complaint of pain which was predominant, severe and prolonged. The then current concept that many pain patients had numerous treatments and complaints (18) and a label of 'chronic pain syndrome' (19) greatly influenced this approach. Pain had to be inconsistent with the anatomic distribution of the nervous system, and

“...no organic pathology or pathophysiological mechanism can account for the pain; or when there is some related organic pathology, the complaint of pain is grossly in excess of what would be expected from the physical findings" (9).

Psychological factors were judged to be etiologically involved in this type of pain, as evidenced by at least one of the following:

\section{1. "A temporal relationship between an environmental stimulus that is apparently related to a psychological conflict or need and the initiation or exacerbation of the pain.}

2. The pain's enabling the individual to avoid some activity that is noxious to him or her.

3. The pain's enabling the individual to get support from the environment that otherwise might not be forthcoming, and

\section{Not due to another mental disorder" (9).}

The discussion of associated features attached to the above criteria emphasizes the tendency of patients to visit physicians frequently, use analgesics excessively, ask too often for surgery and assume an invalid role. This was typical of the contemporary literature (and may still be so in some quarters). Dramatic aspects of the presentation were described. Pain associated with muscle contraction headaches was excluded because of the supposed pathophysiological mechanism accounting for the pain.

Seven years later, DSM-III(R) (10) maintained the category of somatization disorder on the basis of a choice of 13 symptoms from a list of 35 in six groups, of which one was specifically pain symptoms, but four others also included pain. Psychogenic pain became somatoform pain disorder, the possibility of the pain having symbolic features was still mentioned, and in about half the cases, physical trauma was noted to be a predisposing factor. Dramatic pain was again identified. The residual category became Atypical Somatoform Disorder, or Somatoform Disorder Not Otherwise Specified.

\section{Classification of the International Association for the Study of Pain}

The year before the appearance of DSM-III(R), the first edition of the International Association for the Study of Pain (IASP)
Classification of chronic pain: Descriptions of chronic pain syndromes and definitions of pain terms was published (20). In that 1986 edition, as in the later 1994 edition (21), the role of psychological factors, or diagnoses, was carefully defined. Three principal categories of pain of psychological origin were recognized in one overall description. They were muscle tension pain, delusional or hallucinatory pain and hysterical or hypochondriacal pain (Relatively Generalized Syndromes, numbers 1-16 [21]). The type of pain that would correspond most to DSM-III categories of somatization disorder was described under pain of psychological origin: hysterical or hypochondriacal (numbers 1-16, [21]) and it agreed with the DSM-III description in emphasizing frequency of visits to doctors and excessive use of analgesics and other psychotropic drugs without sufficient benefit, while the pain might have a symbolic significance. Three overlapping types were recognized within the subcategory. The first was largely monosymptomatic and related to patients who had pain in one or two regions only, the pain being of fairly recent origin with clear evidence of emotional conflicts, corresponding essentially to somatoform pain disorder of DSM-III(R). The second type of patient had numerous or 'multiple' complaints corresponding approximately to somatization disorder, and a third 'hypochondriacal' subtype was recognized. In the 1986 edition, pain associated with depression was distributed among the above three types. This was done because there did not seem to be a single mechanism for pain associated with depression, even though such pain was frequent. The words 'depressive pain', as indicating a particular type of mechanism, were discouraged.

By 1990, there was increasing dissatisfaction with the DSM-III and DSM-III(R) systems for diagnosing pain disorders on a psychological basis. Somatization disorder was fairly well accepted, hallucinatory pains were extremely rare and hypochondriasis was well understood, but psychogenic pain disorder or somatoform pain disorder presented problems which have still not been wholly resolved. It was widely felt that this category was being used too frequently for conditions that could be better understood as myofascial pain or other organic types of disorders, and that the emphasis on frequent surgery, frequent use of analgesics and frequent consultations was unsatisfactory. It tended to treat as psychological problems, behavioural activities which were quite likely to be consequences of protracted illness rather than evidence of some peculiar personality problem causing the disorder. There was also a school of thought that had originated at the same time as the descriptions of chronic pain disorder (19), which was prepared to consider that chronic pain following a number of injuries or operations might be a result of some anomalous activity in the nervous system, a dysfunction originating at the neuronal level rather than a change triggered by a large variety of possible psychological or psychosocial causes.

Concomitantly, there was a strong behavioural movement in clinical psychology which sought to demonstrate that by treating operant pain (pain that was held to be generated by psychological 'contingencies'), much chronic pain could be relieved.

\section{Changes from DSM-III(R)}

An invited discussion of classification issues in relation to pain and psychiatry was held at the Sixth World Congress of the IASP in Adelaide in 1990. Dissatisfaction was expressed, with particular reference to the tendency to inappropriately use the 
category of "Psychogenic Pain Disorder" in DSM-III(R)and, as the chairperson at the meeting, I was asked from the floor if I would write to the American Psychiatric Association to call for some reform of their system. Accordingly, I wrote to the chairperson of the Task Force, Dr Allen Frances, with respect to possible changes. The request to Dr Frances was courteously received and discussions were undertaken with Dr Robert Spitzer, Dr Steven King and Dr Jim Strain with respect to changes for the better. Subsequently, DSM-IV (11) adopted the arrangement below for what then became "Pain Disorder" without any somatoform or psychogenic prefix, although it still resided within the section on somatoform disorders.

The diagnostic criteria for somatization disorder were concomitantly somewhat simplified, but that was not part of our discussion. The revised criteria for pain disorder became as follows:

1. Pain in one or more anatomical sites was required.

2. The pain caused clinically significant distress or impairment in social, occupational or other important areas of functioning.

3. Psychological factors were judged to have an important role in the onset, severity, exacerbation or maintenance of the pain (Emphasis added).

4. The symptom or deficit was not intentionally produced.

5. The pain was not better accounted for by an affective, anxiety or psychotic disorder.

It is particularly important to note that the exclusionary criterion of a mood, anxiety or psychotic disorder prevented a large number of patients with pain secondary to anxiety or depression from being misclassified in a section intended to reflect a motivational basis for the pain. This had always been present but not so strongly stated in DSM-III and DSM-III(R).

Once the diagnosis for Pain Disorder had been made, it was expected to be diagnosed under two headings. First, Pain Disorder Associated with Psychological Factors was identified and needed to meet the criteria outlined above, while there should be no medical condition of note responsible for the pain. Second, when both psychological factors and a general medical condition were present and promoting the pain, both had to be identified.

DSM-IV underlined two extra considerations. First, Pain Disorder Associated with a General Medical Condition, where the medical condition had a major role in the onset, severity, exacerbation or maintenance of the pain, should have a diagnostic code for the pain selected on the basis of the associated general medical condition, ie, from the International Classification of Diseases (4), or the IASP system.

These criteria limit the excessive use of the diagnosis of Pain Disorder, except where the authors have not followed these conditions.

A subsequent edition of DSM-IV, namely DSM-IV(TR), eg, text revision, did not alter the criteria.

\section{Classification: IASP}

Meanwhile, the second edition of the IASP edition of Classification of Chronic Pain: Descriptions of chronic pain syndromes and definitions of pain terms (21), published in the same year as DSM-IV, maintained its existing distinctions with respect to psychological categories, and added one for depression where pain was associated with depression, and depression commenced first (Code I.16.4 [21, page 56]). This allowed for the cases where pain appeared possibly to originate with depression, typically where a headache emerges with some depressive illnesses and remits with them. Other types of pain associated with depression might be muscle contraction pain, or delusional, hysterical or hypochondriacal pain and could be diagnosed as required under the alternative categories.

The International Classification of Diseases, 10th revision (4) has a similar category to the DSM-IV for persistent somatoform disorder with respect to pain that occurs in association with emotional conflict or psychosocial problems that are sufficient to be the main causative influences. Pain from depressive disorder is excluded. For this category, it is important to make such a diagnosis only on the basis of substantial psychological evidence. The exclusion of organic disorders is not sufficient to warrant the diagnosis, and there must be positive evidence so that if the criteria are followed carefully, this diagnosis will rarely be made.

\section{Avoid 'somatizing and somatization'}

Then what is somatizing? There are a number of current problems with the use of the word somatization or somatizing. About $80 \%$ of healthy individuals experience somatic symptoms in any one week $(22,23)$. Psychological problems affect up to $30 \%$ of patients presenting to physicians in general practice, often with physical symptoms initially $(24,25)$. Thus, physical symptoms, of which the most common is almost always pain, may serve as a route to the family physician or general practitioner, or internist, while the intention is to receive help with the psychological problems. Strong selection processes will further shape the pattern of complaint of patients who are found in pain clinics (26). Those who are conventionally described as 'somatizing' may include patients with high levels of functional somatic distress measured by a somatic symptom index; hypochondriasis measured by high scores on a measure of illness, worry over somatic issues, and the absence of serous illness; or clinical presentations among patients with major depression or anxiety (27). In a sample of 685 patients attending two family medicine clinics, Kirmayer and Robbins found that $26.3 \%$ met the criteria for one or more of the forms of somatization (27). The three forms were associated with different sociodemographic and illness behaviour characteristics, but a majority of the patients met criteria for only one type of somatization, suggesting distinct pathogenic processes may be involved with each of the three types. In any event, almost one half of the 'somatizing' patients were recognized to have significant anxiety or depression that was likely to be causing their complaints.

In clinical practice and in research reports, the term somatization is used in a variety of ways which may be summarized, perhaps incompletely, as follows:

a) Somatization disorder;

b) Conversion symptoms (with pain);

c) Hypochondriasis;

d) Heightened bodily awareness (alerting) resembling 
hypochondriasis, but unlike the latter in responding to reassurance on examination or investigation;

e) Psychophysiological events associated with anxiety or depression

f) Certain types of somatic complaints in schizophrenic patients, perhaps with a delusional basis

(eg, hypochondriacal psychosis); and

g) Any of the above combined with organic disease.

What seems not to be useful for clinical purposes is to talk of patients as somatizing, which automatically implies actively producing physical symptoms, whether indirectly or from some 'unconscious' motive.

This is the point at which social and cultural factors may also play a large part in the selection process and in the corresponding implications as to what is meant by somatizing if it refers to bodily symptoms that may have some psychological function or basis.

Weighty evidence exists that most pain that lacks alleged physical support is actually due to organic factors. This is particularly true with cervical sprain injuries and low back sprain injuries, where so-called 'soft tissue disorders' result in continuing intractable pain in a number of patients. This group is ordinarily a minority and usually constitutes not more than $10 \%$ of all injured patients after three years or more, but despite a lack of readily observable physical signs, tends to

\section{REFERENCES}

1. de Montaigne ME. (1580) Essais. Book I, Chap. 40:374-375. J-V Le Clerc (ed). Paris: Garnier Freres, 1865. (1580) Essais. Book I, Chap. 14. Trans E.J. Trechmann. Oxford University Press, 1927. (N.B. In some editions Chaps. 14 and 40 are transposed).

2. Hodgkiss A. From Lesion to Metaphor: Chronic Pain in British, French and German Medical Writings. 1800-1914. Clio Medica 58. Amsterdam: Rodopi, 2000.

3. Merskey H. The Analysis of Hysteria: Understanding Conversion and Dissociation, 2nd edn. London: Gaskell, 1995.

4. World Health Organization (WHO). International Classification of Diseases, 10th revision. Geneva: WHO, 1992.

5. Breuer J, Freud S. Studies on Hysteria. Complete Psychological Works of Freud, standard edn, vol 2. London: Hogarth Press, 1955.

6. Charcot JM. In: Richer P, ed. Paralysies et Contractures Hystériques. Paris: Octave Doin, 1892.

7. Esterson A. Seductive Mirage: An Exploration of the Work of Sigmund Freud. Chicago: Open Court, 1993.

8. Jones E. Papers on Psychoanalysis. New York: William Wood \& Co, 1913:196.

9. American Psychiatric Association. Diagnostic and Statistical Manual of Mental Disorders, 3rd edn. Washington: American Psychiatric Association, 1980.

10. American Psychiatric Association. Diagnostic and Statistical Manual of Mental Disorders, 3rd edn (revised). Washington: American Psychiatric Association, 1987.

11. American Psychiatric Association. Diagnostic and Statistical Manual of Mental Disorders, 4th edn. Washington: American Psychiatric Association, 1994.

12. Slater E. Diagnosis of 'hysteria'. Br Med J 1965;5447:1395-9.

13. Cohen ME, Robins E, Purtell JJ, Altmann MW, Reid DE. Excessive surgery in hysteria: Study of surgical procedures in 50 women with hysteria and 190 controls. J Am Med Assoc 1953;151:977-86.

14. Guze SB. The diagnosis of hysteria: What are we trying to do? Am J Psychiatry 1967;124:491-8.

15. Mai F, Merskey H. Briquet's concept of hysteria: A historical perspective. Can J Psychiatry 1981;26:57-63.

16. Kellner R. Somatization and Hypochondriasis. New York: Praeger Publishers, 1986.

17. Lipowski ZJ. Somatization: The concept and its clinical application. Am J Psychiatry 1988;145:1358-68. persist with a characteristic pattern. It has been shown in a series of investigations that they often have significant physical problems, eg, cervical facet joint damage $(28,29)$ or intradiscal lesions (30). As well, it is now widely recognized that regional pain syndromes can be, and very probably are, usually, physiological in origin rather than psychological (31).

In these circumstances, it is increasingly dangerous to use the words 'somatizer' or 'somatizing', or any of the somatoform pain disorders, with the possible exception of somatization disorder, which still seems to be a rather restricted category, suitably identified over many years. As for "Pain Disorder", it should only be diagnosed very rarely. When the patient has a psychiatric diagnosis, such as a depressive disorder causing headaches, it should be identified as such. If a physical cause and a 'psychiatric' diagnosis both contribute to the pain, both should be specified as doing so. Any complicating diagnosis not contributing to pain should of course be recognized as well.

These arrangements provide for the recognition of dual or multiple causes of pain - dualism of etiology - and for a monistic view of the pain experience which, at least as a rule, cannot be split up into organic or psychological components.

Someone might ask what all this has to do with the effort to treat patients and to develop an adequate idea of what to do for them. To which the answer is both a platitude and an important principle. It is best to be clear in one's mind about what one is attempting to do for patients if one wishes at all to convey a clear understanding to them in turn.

18. Sternbach RA. Pain patients. Traits and treatment. New York: Academic Press, 1974.

19. Black RG. The chronic pain syndrome. Surg Clin of North Am 1975;55:999-1011.

20. Classification of Chronic Pain: Descriptions of Chronic Pain Syndromes and Definitions of Pain Terms. Monograph for the Subcommittee on Taxonomy, International Association for the Study of Pain, Pain Supplement 3. Merskey H, ed. Amsterdam: Elsevier Science Publishers, 1986.

21. Classification of chronic pain: Descriptions of Chronic pain Syndromes and Definitions of Pain Terms. Monograph for the Task Force on Taxonomy, International Association for the Study of Pain, 2nd edn. Merskey H, Bogduk N, eds. Seattle: International Association for the Study of Pain, 1994.

22. Reidenberg MM, Lowenthal DT. Adverse non-drug reactions. New Engl J Med 1968;279:678-9.

23. Pennebaker JW, Burman MA, Schaeffer MA, Harper DC. Lack of control as a determinant of perceived physical symptoms. J Pers Soc Psychol 1977;35:167-74.

24. Kessell WI. Psychiatric morbidity in a London general practice. Br J Prev Soc Med 1960;14:16-22.

25. Shepherd M, Cooper B, Brown MC, Kalton G. Psychiatric illness in general practice. London: Oxford University Press, 1966.

26. Merskey H, Lau C, Russell ES, et al. Screening for psychiatric morbidity. The pattern of psychological illness and premorbid characteristics in four chronic pain populations. Pain 1987;30:141-57.

27. Kirmayer L, Robbins JM. Three faces of somatization in primary care: Prevalence, concurrence and socio-demographic characteristics. J Nerv Ment Dis 1991;179:647-55.

28. Barnsley L, Lord SM, Bogduk N. Comparative local anaesthetic blocks in the diagnosis of cervical zygapophyseal joint pain. Pain 1993;55:99-106.

29. Lord SM, Barnsley L, Wallis BJ, McDonald GJ, Bogduk N. Percutaneous radiofrequency neurotomy for chronic cervical zygapophyseal joint pain. New Engl J Med 1996;335:1721-6.

30. Schwarzer AC, Aprill CN, Derby R, Fortin J, Kine G, Bogduk N. The prevalence and clinical features of internal disc disruption in patients with chronic low back pain. Spine 1995;20:1878-83.

31. Merskey H. Regional pain is rarely hysterical. Arch Neurol 1988;45:915-8. 


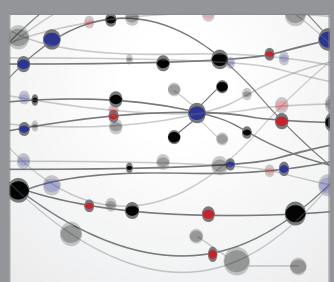

The Scientific World Journal
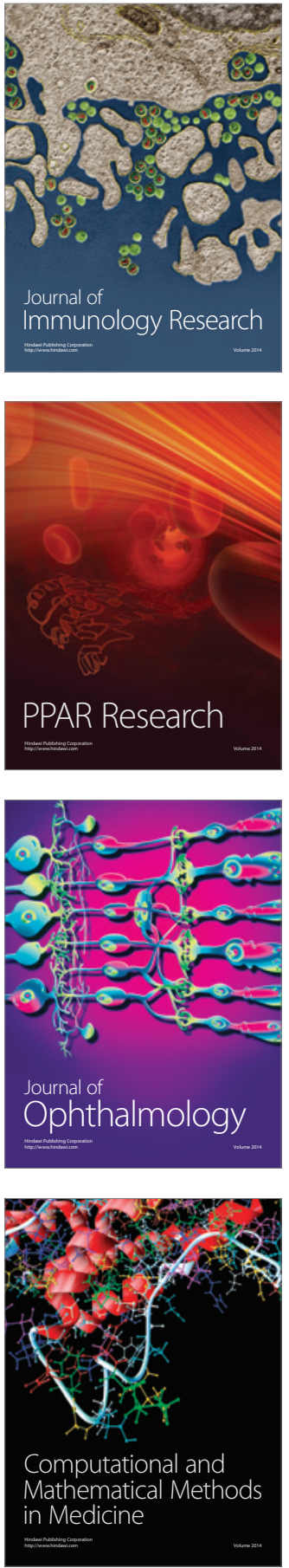

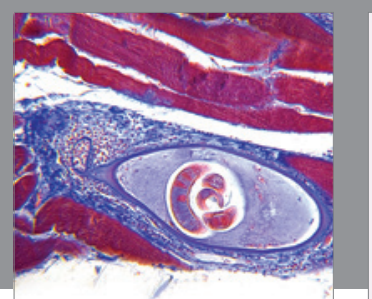

Gastroenterology Research and Practice

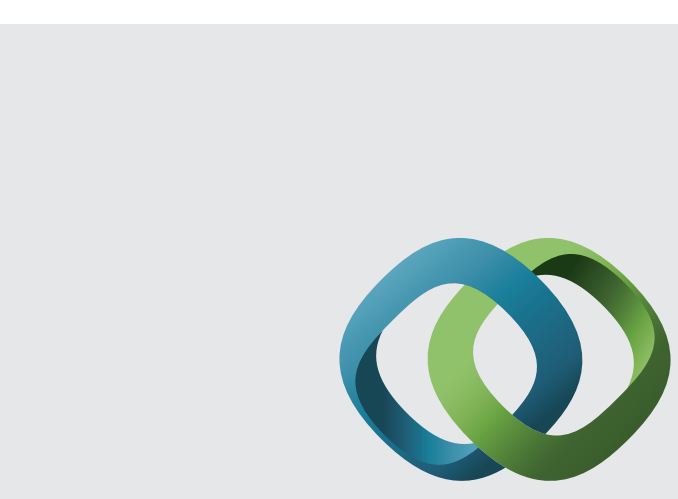

\section{Hindawi}

Submit your manuscripts at

http://www.hindawi.com
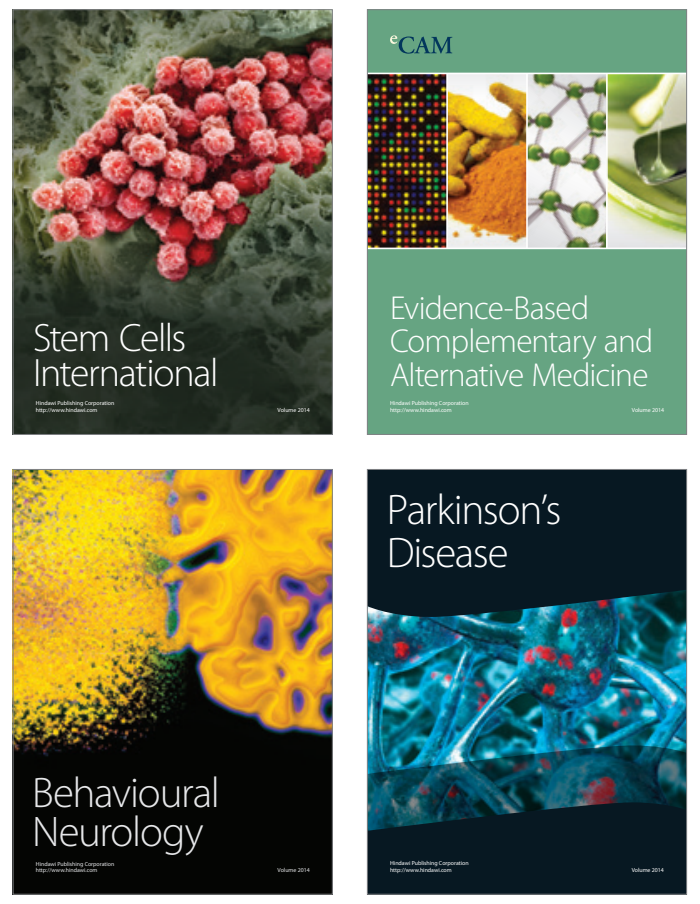
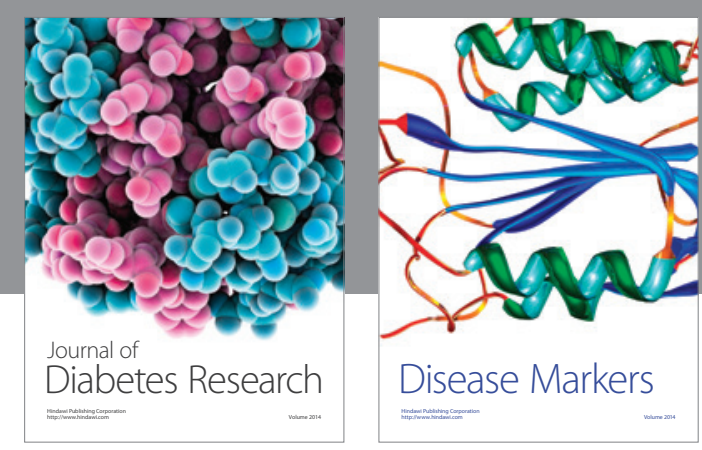

Disease Markers
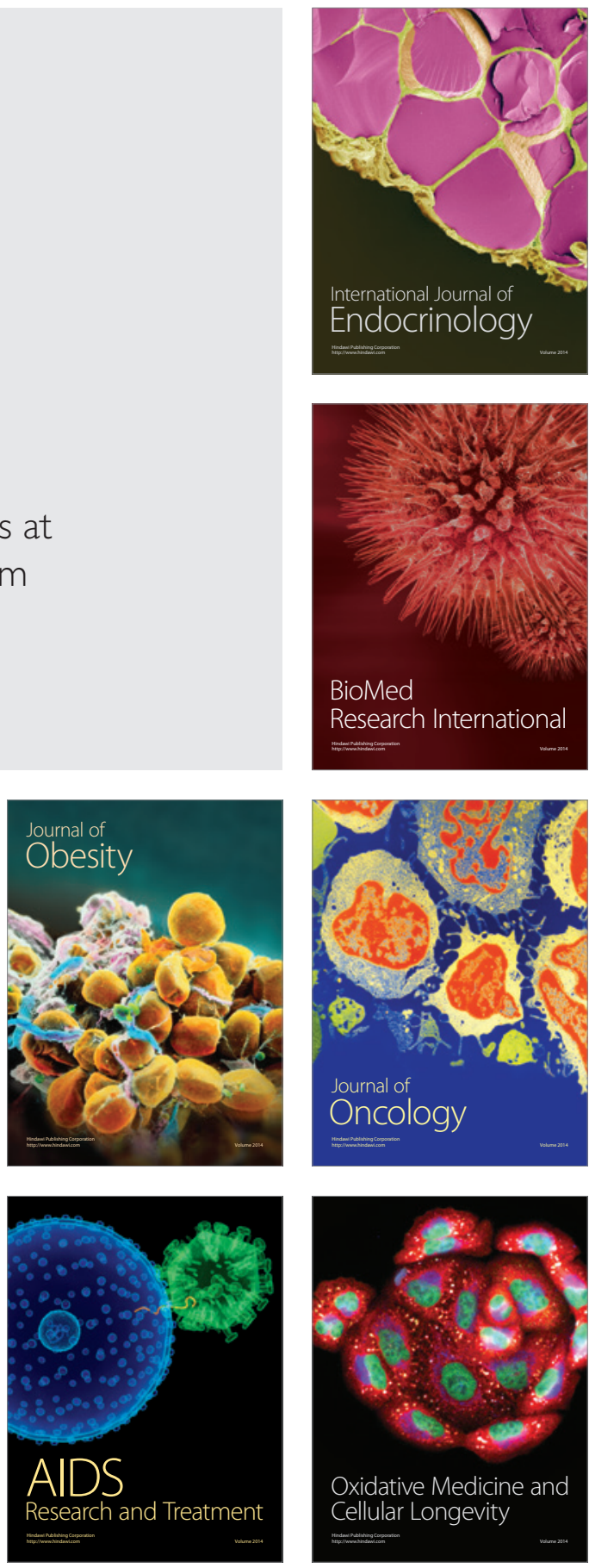